\title{
Sex differences in gout characteristics: tailoring care for women and men
}

\author{
Leslie R. Harrold ${ }^{1 *}$, Carol J. Etzel2,3, Allan Gibofsky ${ }^{4}$, Joel M. Kremer ${ }^{5}$, Michael H. Pillinger ${ }^{6}$, Kenneth G. Saag ${ }^{7}$, \\ Naomi Schlesinger ${ }^{8}$, Robert Terkeltaub ${ }^{9}$, Vanessa Cox $^{2}$ and Jeffrey D. Greenberg ${ }^{2,6}$
}

\begin{abstract}
Background: To characterize the differences between women and men with gout.

Methods: We analyzed a US national cohort of gout patients cared for by rheumatologists.

Results: Compared with the 1012 men with gout, women with gout $(n=262)$ were older $(71$ vs. 61 years, $p<0$. $001)$ and had a greater burden of comorbid conditions $(p<0.001$ for hypertension, diabetes, renal disease and obesity). Risk factors for gout differed with women more often taking diuretics $(p<0.001)$, while men more frequently had dietary triggers $(p<0.05)$.

Conclusions: The profiles of women and men with gout are markedly different, suggesting a need to tailor treatment recommendations.
\end{abstract}

Keywords: Gout, Comorbidity, Gender, Quality of Care

\section{Background}

Gout is the most common inflammatory arthritis, affecting approximately 8.3 million Americans (6.1 million men and 2.2 million women) [1]. Gout prevalence has been increasing over the last half century, due to the aging of the population, increased use of medications that can trigger gout, and the obesity epidemic [2]. Gout is associated with a reduced quality of life, functional impairment, reduced productivity and a higher risk of death [3, 4]. Accumulating evidence suggests that gout is an independent risk factor for cardiovascular morbidity and mortality in both women and men [5].

While gout has been studied for centuries, most research has focused on men. For example, there have been very few studies that have examined the epidemiology of gout based on sex. Those that have included relatively small numbers of women [6, 7] or lacked goutspecific details [8]. Even clinical trials of therapeutic agents for the acute and chronic management of gout included very few women [9-11]. Given the prevalence and impact of gout, it is essential to identify the factors

\footnotetext{
* Correspondence: leslie.harrold@umassmed.edu

1 Department of Medicine and Orthopedics, University of Massachusetts

Medical School, 55 Lake Avenue North, Worcester, MA 01655, USA

Full list of author information is available at the end of the article
}

that impede optimal gout management in both women and men in order to provide tailored treatment recommendations.

In 2012, CORRONA (the Consortium of Rheumatology Researchers of North America) created a national gout registry in which rheumatologists enrolled their gout patients with longitudinal assessment. Within this setting, our objective was to compare women and men with gout to see whether they differed in terms of comorbid conditions associated with gout and risk factors for gout flares. We hypothesized that the patient profile of women with gout would be very different as compared to men with the condition.

\section{Methods}

\section{Data source and population}

CORRONA creates prospective observational cohorts of arthritis patients enrolled by participating rheumatologists in both academic and private practice sites. There were 1273 patients with rheumatologist-diagnosed gout based on the 1977 diagnostic criteria [12] (the registry predated the 2015 American College of Rheumatology Gout Classification criteria) and enrolled in the CORRONA registry database between 11/1/2012 and 3/31/2014 from 34 rheumatology practices across 20 states with 76 
participating rheumatologists. Approvals for data collection and analyses were obtained for academic and private practice sites from local and central institutional review boards, respectively.

\section{Measures and data collection}

Data were collected from patients and their treating rheumatologists using standard clinical research forms based on the American College of Rheumatology [13] treatment recommendations. Information collected included demographics, comorbid conditions, gout presentation, disease severity and activity, family history, body mass index (BMI), dietary intake over the past week, use of medications that can raise serum urate level (e.g., diuretics), use of medications for acute gouty inflammation (nonsteroid anti-inflammatory drugs [NSAIDs], colchicine, steroids) and urate-lowering therapy (ULT) including uricosurics, xanthine oxidase inhibitors, and recombinant uricase [pegloticase]. Documentation included physician exam findings of tophi and inflamed joints, physician and patient global assessments of disease activity, patient assessment of pain, the Health Assessment Questionnaire (HAQ) assessing physical function, and serum urate levels (SUA) from laboratory tests obtained within 10 days of the clinical encounter (this data is not mandated by the study protocol). Patients reported the number of days in the past 3 months there were unable to perform their usual activity. Health care utilization data, including gout hospitalizations within the last 3 years as well as Emergency Room and/or outpatients visits in the past 12 months for gout flares, were gathered. Additionally patients reported how many flares they managed themselves without seeing a health care professional.

\section{Comparison by sex}

We compared patients' baseline characteristics at the time of enrollment, including demographics, goutrelated comorbid conditions and medications, gout characteristics, contributing medical conditions and medications. We compared dietary factors associated with gout flares in men and women in terms of any intake over the past week of beef, pork, seafood and alcohol, as well as the number of weekly servings consumed. We also evaluated rate of use of ULT in the subset of subjects who met ACR criteria for urate lowering (e.g., 2 or more gout flares/year, presence of tophi, history of kidney stones or presence of chronic kidney disease (CKD; stage 3 or greater).

\section{Statistical analysis}

We use a cross-sectional study design, evaluating patients at the time of enrollment. Descriptive statistics were performed using $t$ tests and chi square tests or Fisher exact tests as appropriate. As a sensitivity analysis, when examining the burden of comorbidity between the 2 sexes, we used 3 propensity score (PS) approaches to balance the differences in the ages and disease duration of the women and men with gout. We conducted these sensitivity analyses to address a potential concern that differences in comorbidity burden could have been related to sex differences in age (women were on average 10 years older than men) or disease duration (men had on average 5 more years of gout) The PS approaches allow us to compare women and men with similar demographic profiles. We used 3 approaches to assess the robustness of our findings. For approach \#1, we derived a PS using age and disease duration and used PS trimming to excluded patients with a PS score in the $<5$ percentile and $>95$ percentile range. For approach \#2, we included only those patients who fell inside the area of common support using age and disease duration separately and then used PS trimming. Approach \#3 included only those patients with a conservative common support with PS trimming so that the resulting age and disease duration characteristics of the women and men with gout were within 3 years. All 3 approaches gave similar results to the unadjusted analyses. Given the reduction in the sample size with each of these 3 approaches, we show the unadjusted results in the body of the manuscript but provide the results of the PS approaches in Additional file 1: Table S1. To explore if there were dietary differences between men and women, we conducted adjusted logistic (yes/no dietary intake) and ordinal logistic regression models $(0,1,2+$ servings per week of dietary intake) controlling for age, BMI, duration of gout, comorbidity burden [hypertension, diabetes, renal disease, hyperlipidemia], HCTZ use, other diuretic use and current use of a uratelowering drug. Sex based comparison for functional status was adjusted for age and presented herein. Lastly ULT use was explored in those who met criteria for treatment based on 2 or more attacks in 12 months or presence of tophi (dosages provided in Additional file 2: Table S2).

\section{Results}

There were 262 women and 1011 men enrolled into the registry meeting ACR criteria for gout [12] during the study time period. Women were approximately 10 years older than the men (71.2 vs. $60.9, p<0.0001$; Table 1$)$. Additionally, women were less likely to be white $(88 \%$ vs. $94 \%, p=0.002)$ or to have private insurance $(58 \%$ vs. $75 \%, p<0.001)$. The overall burden of rheumatologist reported comorbid conditions was greater in women in both the overall cohort (Table 1) and PS trimmed subsets (Additional file 1: Table S1). Women were more impaired in terms of days being unable to perform usual work and activities as well as the HAQ score using age adjusted estimates. Activity and HAQ discrepancies persisted after adjusting for comorbidity and duration of gout (Additional file 1: Table S1). Obesity and diuretic 
Table 1 Baseline demographic and gout characteristics

\begin{tabular}{|c|c|c|c|}
\hline \multirow[t]{2}{*}{ Characteristics } & \multirow{2}{*}{$\begin{array}{l}\text { Women } \\
N=262\end{array}$} & \multirow{2}{*}{$\begin{array}{l}\text { Men } \\
N=1011\end{array}$} & \multirow[t]{2}{*}{$P$ value } \\
\hline & & & \\
\hline \multicolumn{4}{|l|}{ Demographics } \\
\hline Age (mean years, SD) & $71.2( \pm 10.7)$ & $60.9( \pm 13.5)$ & $<0.0001$ \\
\hline Race (White n, \%) & $219(84)$ & $897(89)$ & 0.024 \\
\hline Private insurance $(n, \%)$ & $152(58)$ & $757(75)$ & $<0.001$ \\
\hline \multicolumn{4}{|l|}{ Comorbid conditions } \\
\hline BMI (mean, SD) & $33.5( \pm 7.8)$ & $31.9( \pm 6.5)$ & 0.001 \\
\hline Osteoarthritis (n, \%) & $120(46)$ & $256(25)$ & $<0.001$ \\
\hline Other inflammatory arthritis (n,\%) & $19(7)$ & $34(3)$ & $<0.001$ \\
\hline \multicolumn{4}{|l|}{ Medical comorbidities $(n, \%)$} \\
\hline Heart disease & $41(16)$ & $147(14)$ & 0.652 \\
\hline Hypertension & $201(77)$ & $574(57)$ & $<0.001$ \\
\hline Diabetes mellitus & $73(28)$ & $168(17)$ & $<0.001$ \\
\hline Renal disease & $64(24)$ & $136(13)$ & $<0.001$ \\
\hline Hyperlipidemia & $120(46)$ & $394(39)$ & 0.045 \\
\hline Peripheral arterial disease & $2(1)$ & $13(1)$ & 0.485 \\
\hline \multicolumn{4}{|l|}{ Functional Status (mean, SD) } \\
\hline Number of days unable to do usual work/activities in the prior 3 months & $11.1( \pm 23.7)$ & $6.9( \pm 195)$ & 0.003 \\
\hline \multicolumn{4}{|l|}{ Health Assessment Questionnaire } \\
\hline Age adjusted means & $0.59( \pm 0.11)$ & $0.49( \pm 0.13)$ & $<0.001$ \\
\hline \multicolumn{4}{|l|}{ Medications that may trigger gout $(n, \%)$} \\
\hline Aspirin & $76(29)$ & $285(28)$ & 0.794 \\
\hline $\mathrm{HCTZ}$ & $51(20)$ & $105(10)$ & $<0.001$ \\
\hline Diuretics other than HCTZ & $83(32)$ & $116(11)$ & $<0.001$ \\
\hline \multicolumn{4}{|l|}{ Gout characteristics } \\
\hline Family history (n, \%) & $180(69)$ & $682(67)$ & 0.701 \\
\hline Crystal proven (n, \%) & $65(25)$ & $346(34)$ & 0.004 \\
\hline Duration of gout (mean, SD) & $6.1( \pm 7.1)$ & $11.0( \pm 9.8)$ & $<0.0001$ \\
\hline Maximum serum uric acid (mean, SD) & $8.8( \pm 2.3)$ & $8.7( \pm 2.1)$ & 0.481 \\
\hline Current serum uric acid (mean, SD) ${ }^{\mathrm{a}}$ & $5.9( \pm 2.6)$ & $6.0( \pm 1.9)$ & 0.449 \\
\hline Presence of tophi (n, \%) & $60(23)$ & $224(22)$ & 0.791 \\
\hline Contraindications for treatment with: & $11(10)$ & $66(16)$ & 0.0 .170 \\
\hline NSAIDS & $80(31)$ & $206(20)$ & $<0.001$ \\
\hline Colchicine & $32(12)$ & $61(6)$ & 0.001 \\
\hline Flare frequency in prior 12 months (mean, SD) & $3.5( \pm 6.4)$ & $3.7( \pm 8.2)$ & 0.692 \\
\hline \multicolumn{4}{|l|}{ Health care utilization due to gout $(n, \%)$} \\
\hline Proportion of patients with hospitalizations for gout in the past 3 years & $7(3)$ & $19(2)$ & \\
\hline Proportion with an ER visit for a gout flare in the past 12 months & $37(14)$ & $118(12)$ & 0.275 \\
\hline Proportion with an outpatient physician visit for a gout flare in the past 12 months & $124(48)$ & $479(48)$ & 0.918 \\
\hline \multicolumn{4}{|l|}{ Frequency of health care utilization for a flare in the prior 12 months: } \\
\hline ER visits (mean, SD) & $0.32( \pm 1.17)$ & $0.26(1.49)$ & 0.560 \\
\hline Outpatient encounters (mean, SD) & $1.40( \pm 2.64)$ & $1.30( \pm 2.68)$ & 0.613 \\
\hline
\end{tabular}

alaboratory data were available for 215 women and 870 men with gout 
use were also more common in women. Gout characteristics at initial clinical presentation (data not shown) were similar with respect to podagra, monoarthritis, and polyarthritis. Additionally, both the maximum and most recent serum sUA levels were similar, as well as the prevalence of tophi, flare frequency and gout related health care utilization. Women's gout was less likely to be crystal proven $(25 \%$ vs. $34 \%, p=0.004)$. Patterns of dietary intake differed based on sex. Men were more likely to report intake of beef, pork, seafood, beer, wine and hard liquor in unadjusted and adjusted analyses (Fig. 1) as well as a greater number of servings of those items (Additional file 3: Figure S1).

Medications including colchicine, NSAIDs, and glucocorticoids, which can be used either for an acute gouty flare or for prophylaxis when initiating a ULT, were prescribed at similar rates in women and men (Table 2). In addition, over-the-counter supplements for gout were used in both women and men. However, women had greater use of acetaminophen and opioid analgesics. Among those who met criteria for ULT, women were as likely as men to receive it in both unadjusted $(77 \%$ vs. $83 \%, p=0.10)$ or PS adjusted (OR 0.70 , 95\% confidence interval $0.43-1.15$ ) analyses. Women were more likely to receive febuxostat, while men more likely allopurinol (Table 2).

\section{Discussion}

Using a national gout registry, we were able to compare women and men with gout and identify differences in patient profiles with respect to gout-related burden of comorbid disease, contributing medical conditions, medications and diet. Even after using PS trimming techniques to balance the patient populations in terms of age and duration of gout, women had a greater burden of
Table 2 Medications and supplements to treat gout

\begin{tabular}{|c|c|c|c|}
\hline \multirow[t]{2}{*}{ Medication $^{a}$} & Women & Men & \multirow[t]{2}{*}{$P$ value } \\
\hline & $N=262$ & $N=1012$ & \\
\hline \multicolumn{4}{|c|}{ Acute/prophylactic gout medications ( $n, \%)$} \\
\hline Colchicine & $86(33)$ & $366(36)$ & 0.314 \\
\hline Oral steroids & $34(13)$ & $137(14)$ & 0.813 \\
\hline NSAIDs & $63(24)$ & $283(28)$ & 0.204 \\
\hline \multicolumn{4}{|l|}{ Analgesic medications (n, \%) } \\
\hline Acetaminophen & $34(13)$ & $51(5)$ & $<0.001$ \\
\hline Opioid type pain relievers & $40(15)$ & $76(8)$ & $<0.001$ \\
\hline
\end{tabular}

Over the counter supplements for gout (use in the past week) $(n, \%)$

$\begin{array}{llll}\text { Cherry Juice } & 21(8) & 111(11) & 0.161 \\ \text { Vitamin C } & 59(23) & 225(22) & 0.927 \\ \text { Turmeric } & 4(2) & 24(2) & 0.405\end{array}$

Chronic gout medications in those who are candidates

$\begin{array}{llll}\text { Candidates } & 164 & 558 & \\ \text { Any ULT use } & 127(77) & 488(83.0) & 0.103 \\ \text { Allopurinol } & 84(51) & 378(64) & 0.002 \\ \text { Febuxostat } & 42(26) & 95(17) & 0.002\end{array}$

${ }^{\mathrm{a} N S A I D s}$ nonsteroidal anti-inflammatory drugs, ULT urate lowering therapy

musculoskeletal and medical comorbid illness as well as functional impairment. Factors that contribute to gout, including obesity, diuretic medications and diet differed by sex. Women more often had renal disease, and concomitant use of thiazides and/or other diuretics. In contrast, men were more likely to report intake of foods associated with both the development of gout and gout flares, including beef, pork, seafood, and alcohol. The most striking difference was seen in the intake of alcohol. This does reflect national trends in the US that demonstrate that men consume greater quantities of alcohol

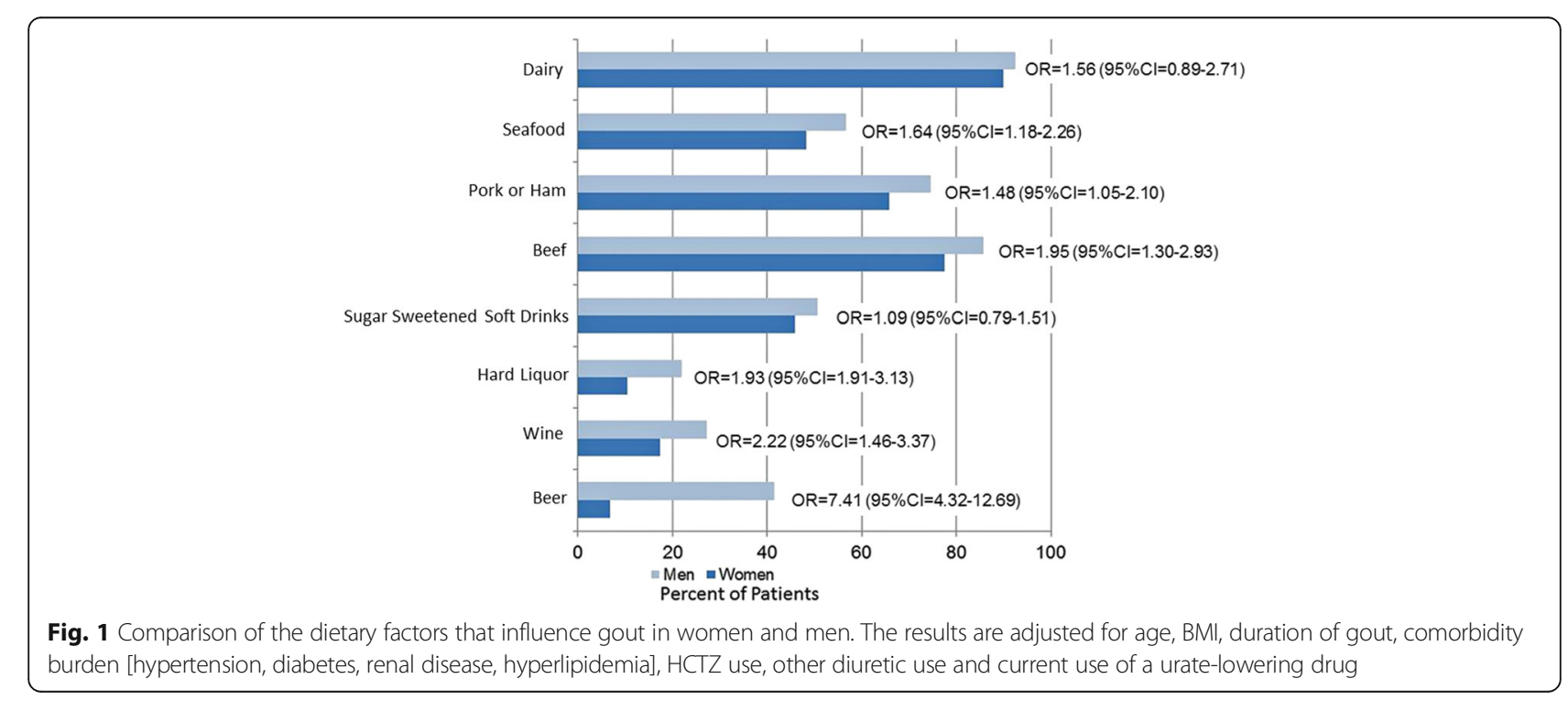


overall as compared to women with beer as the top choice for men while women drinkers have a preference for wine [14].

Our study is similar to others demonstrating women with gout to be older with a greater burden of comorbid illness, and less likely to have their disease proven by crystal diagnosis [6-8]. It is unclear whether lack of crystal confirmation was due to women being less likely offered the procedure versus more likely to decline having it performed. Alternatively, the presentation of women might have been more definitively consistent with the diagnosis of gout and thus the rheumatologist did not pursue further testing. There were differences in the use of anti-inflammatory and analgesic medications, with women were more likely to be receiving acetaminophen and narcotics. However, these medications may have been used for other arthritic or painful conditions.

While patients of both sexes should receive care consistent with "best practices", our observations can help providers to prioritize which factors to explore in terms of contributing to gout in men and women. We anticipate that that patient sex will influence the management of gout. For women, the greater prevalence of increased BMI and diuretic use in this subset suggests this will need to be targeted for intervention. Weight loss has been shown to decrease gout flares and can improve the management of the associated comorbid conditions such as osteoarthritis and diabetes $[15,16]$. For men with gout, a modification of urate-generating dietary intake will need to be pursued given the association with gout flares consistent with treatment recommendations [13, 17-19].

An important strength of this study is that the registry includes rich clinical data on a national sample of gout patients cared for by rheumatologists. Limitations include the study cohort may not be a representative sample of US gout patients, although that seems less likely given the comparisons to other national gout populations [8]. Since this is not an inception cohort, we also could not assess the true baseline characteristics of patients at the time of gout diagnosis. However to address this limitation, we used PS methodology to explore the impact of imbalance in age and disease duration between the sexes and found similar findings.

\section{Conclusions}

In conclusion, this national cohort demonstrates that the gout patient profile differs in women as compared to men. Potentially modifiable factors such as BMI, diuretic use and diet differ by sex. Additionally, the coexistence of comorbidities that influence the management of the condition, such as renal disease and diabetes, are much more common in women as compared to men. A better understanding of sex differences in gout patient profiles will provide a foundation for tailored treatment recommendations by providers.

\section{Additional files}

Additional file 1: Table S1. Baseline characteristics. (DOCX $15 \mathrm{~kb}$ )

Additional file 2: Table S2. Dosing of urate-lowering therapies among eligible patients. (DOCX $12 \mathrm{~kb}$ )

Additional file 3: Figure S1. Comparison of the dietary servings among women and men with gout. The $p$ values represent adjusted analyses adjusting for age, BMI, duration of gout, comorbidity burden [hypertension, diabetes, renal disease, hyperlipidemia], HCTZ use, other diuretic use and current use of a urate-lowering drug. (TIF $320 \mathrm{~kb}$ )

\section{Abbreviations}

BMI: Body mass index; CKD: Chronic kidney disease; CORRONA: Consortium of Rheumatology Researchers of North America; HAQ: Health Assessment Questionnaire; NSAIDs: Nonsteroid anti-inflammatory drugs; PS: Propensity score; SUA: Serum urate levels; ULT: Urate-lowering therapy

\section{Acknowledgements}

The authors wish to thank Leslie Whitehead for her editorial assistance with the manuscript. In addition, we thank the participating rheumatologists and their patients contributing data to the Corrona Gout Registry.

\section{Funding}

In the last 2 years, AbbVie, Amgen, AstraZeneca, Bristol-Myers Squibb, Crescendo, Eli Lilly, Genentech, Horizon Pharma USA, Janssen, Novartis, Pfizer, and UCB have supported Corrona, LLC through contracted subscriptions. The study design, data analysis and reporting of results in this manuscript were performed independent of all funding sources. One of the investigators received support from the National Institutes of Health (AR053856: LRH).

\section{Availability of data and material}

The data that support the findings of this study are available through Corrona, LLC, but restrictions apply to the availability of these data, which were used under a subscription agreement for the current study, and so are not publicly available.

\section{Authors' contributions}

LRH and CJE had full access to all of the data in the study and take responsibility for the integrity of the data and the accuracy of the data analysis. All authors were involved in the design and conduct of the study; collection, management, analysis, and interpretation of the data; and preparation, drafting, critical review, and approval of all versions of the manuscript, its content, and its submission to BMC Musculoskeletal Disorders. All authors take full public responsibility for the manuscript content.

\section{Competing interests}

LR Harrold: Corrona, Genentech, Pfizer, AstraZeneca; CJ Etzel and V Cox: Corrona; A Gibofsky: AbbVie, Amgen, BMS, Celgene, Drais, GSK, Horizon Pharma, Iroko, Johnson \& Johnson, Medac, Pfizer, Relburn, Samumed, Takeda; JM Kremer: Corrona, AbbVie, Amgen, BMS, Genentech, Lilly, Pfizer; M Pillinger: Takeda, Savient; K Saag: Takeda, Ardea; N Schlesinger: Novartis, Takeda, Sobi, Pfizer, Astra Zeneca; R Terkeltaub: VA Research Service. NIH. ARDEA, Sobi, Selecta, Aequus, Horizon, Revive, Relburn, SOBI; VCox: Corrona; JD Greenberg: Corrona, Astra Zeneca, Genentech, Janssen, Celgene, Novartis, Pfizer.

\section{Consent for publication}

All the results presented in the manuscript are in aggregate form and no personal identifiable information was used for this study.

\section{Ethics approval and consent to participate}

All participating investigators were required to obtain full board approval for conducting research involving human subjects. Sponsor approval and continuing review was obtained through a central IRB (New England Independent Review Board, NEIRB No. 02-021). For academic investigative 
sites that did not receive a waiver to use the central IRB, full board approva was obtained from the respective governing IRBs (noted below) and documentation of approval was submitted to the Sponsor prior to initiating any study procedures.

082 - Northwestern University Chicago IL Gout

054 - University of Rochester Rochester NY Gout

127 - Montefiore Medical Center Bronx NY Gout

All registry subjects were required to provide written informed consent prior to participating.

\section{Publisher's Note}

Springer Nature remains neutral with regard to jurisdictional claims in published maps and institutional affiliations.

\section{Author details}

${ }^{1}$ Department of Medicine and Orthopedics, University of Massachusetts Medical School, 55 Lake Avenue North, Worcester, MA 01655, USA. Corrona, LLC, Southborough, MA, USA. ${ }^{3}$ Department of Epidemiology, UT MD Anderson Cancer Center, Houston, TX, USA. ${ }^{4}$ Hospital for Special Surgery-Weill Medical College of Cornell University, New York, NY, USA. ${ }^{5}$ Albany Medical College and The Center for Rheumatology, Albany, NY, USA. ${ }^{6} \mathrm{NYU}$ School of Medicine, New York, NY, USA. ${ }^{7}$ University of Alabama at Birmingham, Birmingham, AL, USA. ${ }^{8}$ Rutgers-Robert Wood Johnson Medical School, New Brunswick, NJ, USA. 'VA Medical Center, UCSD, San Diego, CA, USA.

Received: 3 October 2016 Accepted: 1 March 2017

Published online: 14 March 2017

\section{References}

1. Zhu Y, Pandya BJ, Choi HK. Prevalence of gout and hyperuricemia in the US general population: the National Health and Nutrition Examination Survey 2007-2008. Arthritis Rheum. 2011;63:3136-41.

2. Bieber JD, Terkeltaub RA. Gout: on the brink of novel therapeutic options for an ancient disease. Arthritis Rheum. 2004;50:2400-14.

3. Singh JA, Strand V. Gout is associated with more comorbidities, poorer health-related quality of life and higher healthcare utilisation in US veterans. Ann Rheum Dis. 2008;67:1310-6.

4. Edwards NL, Sundy JS, Forsythe A, Blume S, Pan F, Becker MA. Work productivity loss due to flares in patients with chronic gout refractory to conventional therapy. J Med Econ. 2011;14:10-5.

5. Krishnan E, Baker JF, Furst DE, Schumacher HR. Gout and the risk of acute myocardial infarction. Arthritis Rheum. 2006;54:2688-96.

6. Lally EV, Ho Jr G, Kaplan SR. The clinical spectrum of gouty arthritis in women. Arch Intern Med. 1986;146:2221-5.

7. Puig JG, Michan AD, Jimenez ML, Perez de Ayala C, Mateos FA, Capitan CF, et al. Female gout. Clinical spectrum and uric acid metabolism. Arch Intern Med. 1991;151:726-32.

8. Harrold LR, Yood RA, Mikuls TR, Andrade SE, Davis J, Fuller J, et al. Sex differences in gout epidemiology: evaluation and treatment. Ann Rheum Dis. 2006;65:1368-72

9. Terkeltaub RA, Schumacher HR, Carter JD, Baraf HS, Evans RR, Wang J, et al. Rilonacept in the treatment of acute gouty arthritis: a randomized, controlled clinical trial using indomethacin as the active comparator. Arthritis Res Ther. 2013;15:R25.

10. Sundy JS, Baraf HS, Yood RA, Edwards NL, Gutierrez-Urena SR, Treadwell EL, et al. Efficacy and tolerability of pegloticase for the treatment of chronic gout in patients refractory to conventional treatment: two randomized controlled trials. JAMA. 2011;306:711-20.

11. Becker MA, Schumacher Jr HR, Wortmann RL, MacDonald PA, Palo WA, Eustace D, et al. Febuxostat, a novel nonpurine selective inhibitor of xanthine oxidase: a twenty-eight-day, multicenter, phase II, randomized, double-blind, placebo-controlled, dose-response clinical trial examining safety and efficacy in patients with gout. Arthritis Rheum. 2005;52:916-23.

12. Wallace SL, Robinson H, Masi AT, Decker JL, McCarty DJ, Yu TF. Preliminary criteria for the classification of the acute arthritis of primary gout. Arthritis Rheum. 1977;20:895-900.

13. Khanna D, Khanna PP, Fitzgerald JD, Singh MK, Bae S, Neogi T, et al. 2012 American College of Rheumatology guidelines for management of gout. Part 2: therapy and antiinflammatory prophylaxis of acute gouty arthritis. Arthritis Care Res (Hoboken). 2012;64:1447-61.
14. Saad L. http://www.gallup.com/poll/156770/majority-drink-alcoholaveraging-four-drinks-week.aspx. Gallup Poll; 2012. Accessed 10 Mar 2017.

15. Johnston CS, Tjonn SL, Swan PD. High-protein, low-fat diets are effective for weight loss and favorably alter biomarkers in healthy adults. J Nutr. 2004; 134:586-91.

16. Dessein $\mathrm{PH}$, Shipton EA, Stanwix $\mathrm{AE}$, Joffe BI, Ramokgadi J. Beneficial effects of weight loss associated with moderate calorie/carbohydrate restriction, and increased proportional intake of protein and unsaturated fat on serum urate and lipoprotein levels in gout: a pilot study. Ann Rheum Dis. 2000;59: 539-43.

17. Zhang Y, Chen C, Choi H, Chaisson C, Hunter D, Niu J, et al. Purine-rich foods intake and recurrent gout attacks. Ann Rheum Dis. 2012;71:1448-53.

18. Zhang Y, Woods R, Chaisson CE, Neogi T, Niu J, McAlindon TE, et al. Alcohol consumption as a trigger of recurrent gout attacks. Am J Med. 2006;119:800 e13-8.

19. Richette P, Doherty M, Pascual E, Barskova V, Becce F, Castaneda-Sanabria J, et al. 2016 updated EULAR evidence-based recommendations for the management of gout. Ann Rheum Dis. 2017;76:29-42

\section{Submit your next manuscript to BioMed Central and we will help you at every step:}

- We accept pre-submission inquiries

- Our selector tool helps you to find the most relevant journal

- We provide round the clock customer support

- Convenient online submission

- Thorough peer review

- Inclusion in PubMed and all major indexing services

- Maximum visibility for your research

Submit your manuscript at www.biomedcentral.com/submit
) Biomed Central 